\title{
Acanthosis nigricans in obese women with the polycystic ovary syndrome: disease spectrum not distinct entity
}

\author{
Gerard S. Conway and Howard S. Jacobs \\ Cobbold Laboratories, The Middlesex Hospital, Mortimer Street, London WIN 8AA, UK.
}

\begin{abstract}
Summary: Fasting and stimulated insulin concentrations in four patients with acanthosis nigricans and polycystic ovary syndrome were compared with four patients, matched for weight and ovarian morphology, without acanthosis. The median fasting serum insulin concentrations were $\mathbf{1 1 4 . 2}$ and $\mathbf{2 5 . 1}$ $\mathrm{mU} / \mathrm{l}$ in the respective groups $(P=0.02)$. One additional patient was investigated before and after an $18 \%$ increase in weight which resulted in a $170 \%$ increase in fasting insulin concentrations and the development of acanthosis nigricans. These observations suggest that there is variation between individuals in the degree of obesity that results in the development of acanthosis and that obese patients with this skin condition represent the severe end of the spectrum of the polycystic ovary syndrome.
\end{abstract}

\section{Introduction}

Acanthosis nigricans, when not related to malignancy, is considered to be a dermal manifestation of hyperinsulinaemia..$^{1-3}$ The extent to which obesity contributes to insulin resistance in these circumstances is disputed. Several reports have shown that patients with acanthosis nigricans have higher serum insulin concentrations than weight matched subjects without acanthosis, suggesting that their hyperinsulinaemia is not solely caused by obesity. ${ }^{4.5}$ However, the high levels of circulating insulin associated with acanthosis nigricans have fallen with short term dietary restriction, suggesting that obesity may contribute to the hyperinsulinaemia. ${ }^{6}$ The presence of polycystic ovaries, which may also be associated with insulin resistance and acanthosis nigricans, ${ }^{7}$ has not been carefully identified in previous studies. We describe here a patient whose metabolic status was assessed before and after weight gain and the appearance of acanthosis nigricans. The results from this patient are compared with those from obese patients with and without acanthosis.

\section{Case report}

EH presented at the age of 27 with hirsutism and oligomenorrhoea, having been investigated and treated extensively for obesity with very low calorie diets, psychoanalysis, jaw wiring and a gastric balloon, each with only temporary success. Her menarche was at the age of 13 from which time her

Correspondence: G.S. Conway, M.R.C.P. Accepted: 8 February 1990 menstrual cycle was irregular until she received the oral contraceptive at the age of 15 . At 23 years she weighed $86 \mathrm{~kg}$ and conceived without difficulty and had a full term normal delivery. She subsequentlyo became more obese and hirsute. Her paternal family had a history of obesity and hirsutism but her mother and two sisters were of normal build, non-hirsute and had regular menstrual cycles.

On examination she weighed $131.5 \mathrm{~kg}$ and her height was $1.71 \mathrm{~m}$. She was hirsute, euthyroid, normotensive and without evidence of acanthosis nigricans. Investigations showed her to have polycystic ovaries on ultrasound with a normal endocrine profile (Table I). She was treated with spironolactone for 4 months before menorrhagia necessitated its withdrawal.

One year later she weighed $155 \mathrm{~kg}$ and on examination had the new finding of acanthosis nigricans affecting her neck and axillae.

\section{Materials and methods}

Four patients with acanthosis nigricans, all found to have polycystic ovaries on ultrasound, were matched by weight to non-acanthotic patients with polycystic ovaries. A $75 \mathrm{~g}$ oral glucose tolerance test was performed on all subjects after 3 days of a diet containing at least $300 \mathrm{~g}$ of carbohydrate daily and after an overnight fast. Samples of arterialized venous blood were drawn at 15 minute intervals for 3 hours from the dorsum of a warmed hand for measurements of glucose and insulin concentrations. Plasma glucose concentrations were measured immediately by the glucose oxidase method and serum insulin concentrations were 
Table I Results from Case EH before and after the appearance of acanthosis nigricans and the median (range) measurements from subjects with $(n=4)$ and without $(n=4)$ acanthosis

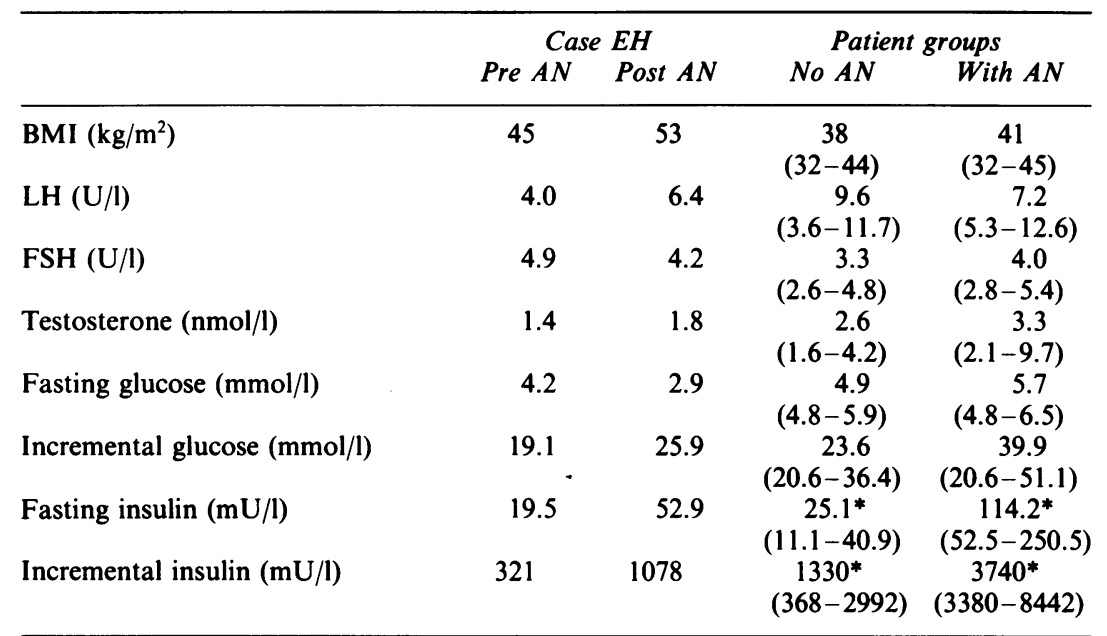

${ }^{*} P=0.02$, Mann-Whitney $\mathrm{U}$ test. $\mathrm{AN}=$ acanthosis nigricans; $\mathrm{BMI}=$ body mass index; $\mathrm{LH}=$ luteinizing hormone; $\mathrm{FSH}=$ follicle stimulating hormone.

measured later by radioimmunoassay. ${ }^{8}$ Serum gonadotrophin and testosterone measurements were performed as previously described. ${ }^{9}$ The body mass index (BMI) was calculated from the equation: $\mathrm{BMI}=$ weight $/ \mathrm{height}^{2}\left(\mathrm{~kg} / \mathrm{m}^{2}\right)$. Incremental areas for glucose and insulin were calculated from the sum of each post-ingestion measurement less the fasting concentration.

\section{Results}

The endocrine results of case EH before and after the appearance of acanthosis nigricans are shown in Table I, together with the median BMI and endocrine profiles from the groups with and without acanthosis. The results from case $\mathrm{EH}$ after the emergence of acanthosis follow closely the differences between the patients with and without established acanthosis (Table I). The group with acanthosis have significantly higher fasting and glucose stimulated serum insulin concentrations and a higher glucose tolerance curve compared with the non-acanthosis group. Figure 1 shows the glucose tolerance curves in patient $\mathrm{EH}$ and in the acanthosis and non-acanthosis groups and demonstrates a deterioration in glucose tolerance in association with this skin condition.

The insulin responses in the glucose tolerance tests in patient $\mathbf{E H}$ are shown in comparison with the acanthosis and non-acanthosis groups (Figure 2 ). While there is a marked increase in the insulin concentrations with the appearance of acanthosis

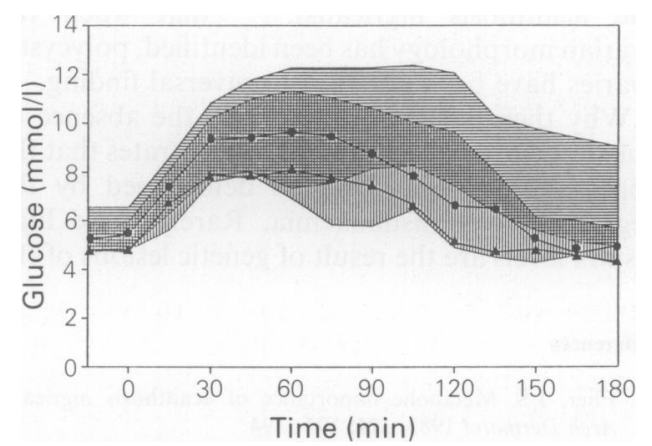

Figure 1 Oral glucose tolerance curves in patient $\mathbf{E H}$ before (triangles) and after (circles) the development of AN compared with the ranges of glucose measurements in the acanthosis (horizontal shading) and nonacanthosis (vertical shading) groups.

in patient $\mathrm{EH}$, there is no clear separation between the insulin responses to glucose from the two groups of patients except for the last 60 minutes of the test when the insulin measurements in subjects with established acanthosis show little sign of returning to basal concentrations.

\section{Discussion}

The initial clinical presentation of case $\mathrm{EH}$ was with the classical features of the polycystic ovary syndrome: oligomenorrhoea, hirsutism and obesity. Subsequent weight increase was associated with the 


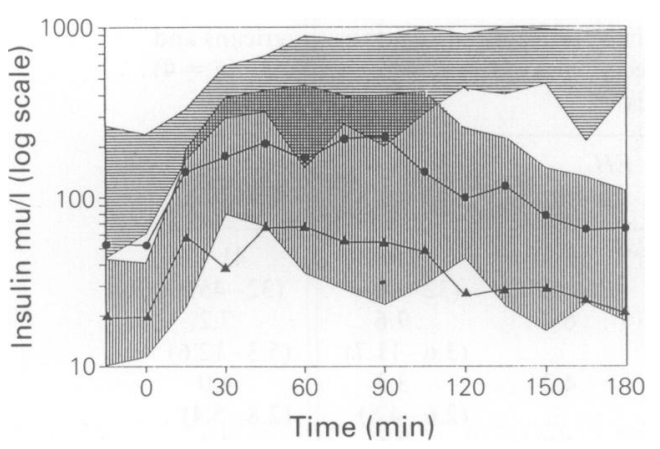

Figure 2 Insulin concentrations during oral glucose tolerance tests in patient EH before (triangles) and after (circles) the development of AN compared with the ranges of insulin measurements in the acanthosis (horizontal shading) and non-acanthosis (vertical shading) groups.

development of acanthosis nigricans. These events led us to question whether other women with acanthosis nigricans have underlying polycystic ovary syndrome. It is evident from the literature of women with acanthosis, including the HAIR-AN syndrome (hyperandrogenism, insulin resistance and acanthosis nigricans), ${ }^{10-14}$ that when the ovarian morphology has been identified, polycystic ovaries have been an almost universal finding.

Why then is acanthosis rare in the absence of polycystic ovaries? Case EH demonstrates that the appearance of acanthosis is determined by the degree of hyperinsulinaemia. Rarely these high insulin levels are the result of genetic lesions of the

\section{References}

1. Flier, J.S. Metabolic importance of acanthosis nigricans Arch Dermatol 1985, 121: 193-194.

2. Matsuoka, L.Y., Wortsman, J., Gavin, J.R. \& Goldman, J. Spectrum of endocrine abnormalities associated with acanthosis nigricans. Am J Med 1987, 83: 719-725.

3. Geffner, M.E. \& Golde, D.W. Selective insulin action on skin, ovary and heart in insulin resistant states. Diabetes Care 1988 , 11: $500-505$.

4. Stuart, C.A., Peters, E.J., Prince, M.J., Richards, G., Cavallo, A. \& Meyer, W.J. Insulin resistance with acanthosis nigricans: the roles of obesity and androgen excess. Metabolism 1986, 35: $197-205$.

5. Peters, E.J., Stuart, C.A. \& Prince, M.J. Acanthosis nigricans and obesity: acquired and intrinsic defects in insulin action. Metabolism 1986, 35: 807-813.

6. Chang, R.J., Nakamura, R.M., Judd, H.L. \& Kaplan, S.A Insulin resistance in nonobese patients with polycystic ovarian disease. J Clin Endocrinol Metab 1983, 57: 356-359.

7. Morgan, C.R. \& Lazarow, A. Immunoassay of insulin: two antibody systems. Diabetes 1963, 120: 115-126.

8. Conway, G.S., Honour, J.W. \& Jacobs, H.S. Heterogeneity of the polycystic ovary syndrome: clinical, endocrine and ultrasound features in 556 patients. Clin Endocrinol 1989, 30: 459-470.

9. Kahn, C.R., Flier, J.S., Bar, R.S. et al. The syndromes of insulin resistance and acanthosis nigricans. $N$ Engl $J$ Med 1976, 294: 739-745. insulin pathway. ${ }^{9-11}$ More commonly, obesitymediated insulin resistance contributes to the hyperinsulinaemia. It may be that obesity, which alone does not result in sufficiently high insulin levels to cause acanthosis, has to occur in the presence of another insulin resistant state (the polycystic ovary syndrome) for the skin effect to be manifest.

The metabolic consequences of obesity are demonstrated here by case $\mathrm{EH}$ in whom an $18 \%$ increase in weight resulted in a $170 \%$ increase in the fasting serum insulin concentration and the development of acanthosis nigricans. Obesitymediated insulin resistance can therefore be critical in the aetiology of acanthosis nigricans. There is clearly a variation between individuals in the threshold at which hyperinsulinaemia leads to this skin change and in the degree of insulin resistance achieved for a given weight. Comparisons of weight matched subjects with and without acanthosis nigricans ${ }^{15}$ simply selects groups with differing insulin sensitivity but provides no information on the cause of the hyperinsulinaemia.

In conclusion, women with acanthosis nigricans and obesity represent the severest form of the polycystic ovary syndrome. The spectrum ranges from lean patients with polycystic ovary syndrome and normal insulin secretion, through lean, mildly hyperinsulinaemic and obese severely hyperinsulinaemic polycystic ovary syndrome to those with acanthosis nigricans. Whereas progression through the severe end of this range may be regulated by obesity, the factors which determine the mild end of the spectrum remain obscure.

10. Leme, C.E., Wajchenberg, B.L., Lerario, A.C., Goldman, J. \& Borges, J.L.C. Acanthosis nigricans, hirsutism, insulin resistance and insulin receptor defect. Clin Endocrinol 1982 , 17: 43-49.

11. Bar, R.S., Muggeo, M., Roth, J., Kahn, C.R., Havrankova, J. \& Imperato-McGinley, J. Insulin resistance, acanthosis nigricans, and normal insulin receptors in a young woman: evidence for a postreceptor defect. J Clin Endocrinol Metab 1978, 47: 620-625.

12. Barbieri, R.L. \& Ryan, K.J. Hyperandrogenism, insulin resistance, and acanthosis nigricans syndrome: a common endocrinopathy with distinct pathophysiologic features. $\mathrm{Am} \mathrm{J}$ Obstet Gynecol 1983, 147: 90-101.

13. Barbieri, R.L., Smith, S. \& Ryan, K.J. The role of hyperinsulinemia in the pathogenesis of ovarian hyperandrogenism. Fertil Steril 1988, 50: 197-212.

14. Flier, J.S., Eastman, R.C., Minaker, K.L., Matteson, D. \& Rowe, J.W. Acanthosis nigricans in obese women with hyperandrogenism. Characterization of an insulin-resistant state distinct from the type A and B syndromes. Diabetes 1985, 34: 101-107.

15. Grigorescu, F., Flier, J.S. \& Kahn, C.R. Characterization of binding and phosphorylation defects of erythrocyte insulin receptors in the type $A$ syndrome of insulin resistance. Diabetes 1986, 35: 127-138. 